\title{
A putative cellular receptor for dengue viruses
}

\author{
The finding that dengue viruses use heparan sulfate to enter cells may alter our understanding of \\ their pathogenesis and aid in vaccine development (pages 866-871).
}

D ENGUE IS AN acute viral disease that affects over 100 million people worldwide. Because there are no vaccines or antivirals available, this disease still offers a great challenge to medicine and molecular biology $y^{1,2}$. A survey of the scientific literature or popular press will convince readers of the seriousness of the problem, hence, the significance of the report in this issue by Chen and colleagues ${ }^{3}$ on the identification of the putative virus receptor.

Of fundamental importance for developing effective strategies to combat dengue is a thorough understanding of the biology and pathogenesis of the virus. Far too little is known about virus-mediated

\section{J. Robert PutNak, NiRANJAN} KANESA-THASAN \& BRUCE L. INNIS

events that cause dengue fever, let alone its more severe manifestations, dengue hemorrhagic fever and shock syndrome. Toward this goal Chen et al. address the first event required for productive infection, namely, attachment of dengue virus (DV) to a susceptible host cell.

Having previously demonstrated that a recombinant DV envelope glycoprotein (E) binds specifically to target cells ${ }^{4}$, these authors set out to identify the cellular receptor for DV. For this study, they chose to focus upon a family of molecules

\section{Possible initial interactions of dengue virus (DV) with the cell.}

a. Initial binding mediated by heparan sulfate (HS).

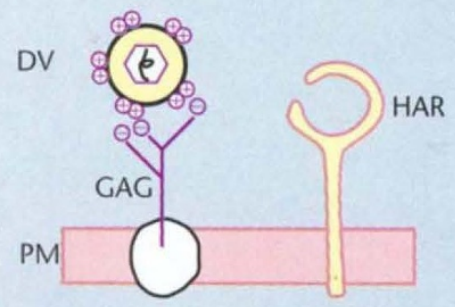
b. Transfer of DV to high affinity receptor (HAR),
triggering endocytosis

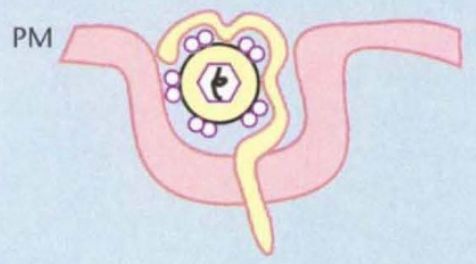

c. Formation of endocytic vacuole and membrane fusion to release nucleocapsid and then RNA.

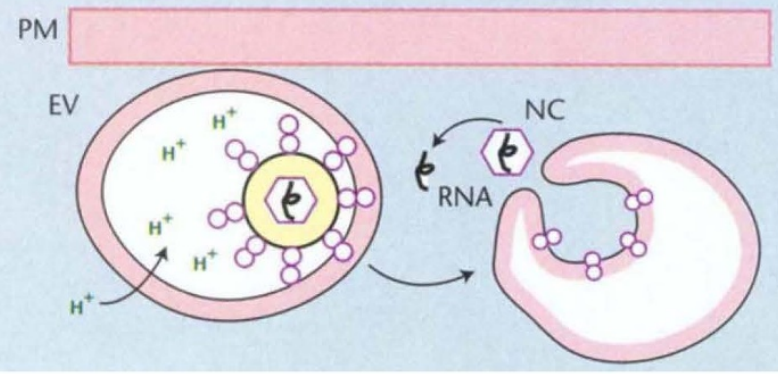

called glycosaminoglycans (GAGs) that are complex, charged carbohydrates found both on the cell surface and in the extracellular matrix. Associated with proteins, they comprise the family of macromolecules called proteoglycans. They are involved in cell-cell interactions and cell proliferation ${ }^{5}$. In addition, they appear to have receptor-like activity for certain biologically active substances such as growth factors, binding them selectively for transport across the basement membrane $^{6}$. Among the most ubiquitous GAGs are the heparan sulfates (HS), present in all tissues and differing only in complexity and degree of sulfation. It is no surprise then, that HS are used by pathogenic microorganisms as diverse as viruses (HIV, human herpes virus, cytomegalovirus and foot and mouth disease virus, FMDV), protozoa and some bacteria (Listeria, Neisseria) to gain entry into host cells .

In a series of well-controlled experiments, Chen and co-workers demonstrate that DV serotype-2 binds selectively to highly-sulfated heparan sulfates (hsHS) on target cells via the viral E glycoprotein. Using as their model the crystal structure of $\mathrm{E}$ protein from a related flavivirus, tick-borne encephalitis virus $^{8}$, they suggest that sites rich in basic amino acids, within domains I and III, and adjacent to an arg-gly-asp (RGD) sequence motif, mediate virus-cell attachment. They further postulate that differences in HS structure and sulfation among cells may account for the selectivity of DV infection. Although opening the door to understanding the complex interactions of DVs with their host cell, this work also raises many new questions.

There are four serotypes of DV, all of which are important in human disease. Whether HS are used by all four serotypes to bind to cells of the host has yet to be demonstrated. Therefore, it is important that these findings be confirmed for the other virus serotypes, although sequence homology within the putative binding domain of $\mathrm{E}$ protein suggests that HS probably bind all flaviviruses. Also unclear is whether HS are the only receptor molecules required for penetration of $\mathrm{DV}$ into the cell. An alter- 
native hypothesis is that HS serve primarily to concentrate virus particles at the cell surface for subsequent interaction with other receptor molecules, most plausibly, members of the integrin family. FMDV interacts with cells in this manner; its initial association with $\mathrm{HS}$ is followed by RGD motif-mediated binding to the integrin $\alpha_{v} \beta_{3}$ (ref. 7). Unlike FMDV, however, DV is enveloped and enters the cell by receptor-mediated endocytosis followed by fusion with the endocytic vacuole (see figure). Nevertheless, a similar role for the RGD motif of DV might be assessed experimentally by site-directed mutagenesis of one of the available DV infectious cDNA clones.

There is an alternative mode of entry of DV into cells that is mediated by antibody-Fc receptor (FcR) interaction, which is strongly implicated in the pathogenesis of more severe disease in individuals with pre-existing antibody. Nothing is known about the involvement of HS in this apparently independent pathway. One feature of DV infection of macrophages by the antibody-FcR-mediated route is the elaboration of interferon- $\gamma$, which causes increased expression of $\mathrm{FCR}$ on nearby cells ${ }^{10}$, thus priming them for infection. This raises the question as to whether HS-mediated viral entry may similarly enhance HS expression in neighboring cells leading to increased numbers and types of virus-susceptible cells.

The speculation by Chen et al. on the possible role of tissue-specific variation in $\mathrm{HS}$ in determining virus tropism is an intriguing one. It is currently believed that DV principally targets macrophages and to an uncertain extent B lymphocytes, bone marrow stromal cells, and hepatocytes. However, the types of HS and levels of expression on these cells and on cells thought to be less susceptible have not been well studied. The types of GAGs present on various cell types, both before and after activation, might be correlated with efficiency of virus infection. Such expression might significantly alter the course of disease.

In addition to opening new avenues for research on DV pathogenesis, the Chen findings may have practical applications. Most immediate is their demonstration that the polysulfated compound suramin, an old and wellstudied antimicrobial, can inhibit DV attachment and infection. The testing of this compound as an antiviral in animal models seems warranted. Moreover, genetic modification of the putative hsHS-binding region of the E glycoprotein gene of DV may provide a direct approach for attenuating the virus for use in live vaccines. Dengue and DV have been the subject of scientific investigation for more than 50 years and many important findings have emerged; cer- tainly, the elucidation of the cellular receptor must rank among these.

1. Halstead, S.B. Pathogenesis of dengue: Challenges to molecular biology. Science 239, 476-481 (1988)

2. Putnak, J.R. Progress in the development of recom binant vaccines against dengue and other arthropod-borne flaviviruses. In Modern Vaccinology (ed. Kurstak, E.) 231-252 (Plenum, New York, 1994).

3. Chen, $Y$. et al. Dengue virus infectivity depends on envelope protein binding to target cell heparan sulfate. Nature Med. 3, 866-871 (1997).

4. Chen, Y., Maguire, T. \& Marks, R.M. Demonstration of binding of dengue virus envelope protein to target cells. J. Virology 70, 8765-8772 (1996)

5. Giuffre, $L$ et al Monocyte adhesion to activated aortic endothelium: role of L-selectin and heparan sulfate proteoglycans.J Cell Biol. 136, 945-956 (1997)

6. Friedl, A., Chang, Z., Tierney, A. \& Rapraeger, A.C Differential binding of fibroblast growth factor-2 and -7 to basement membrane heparan sulfate: comparison of normal and abnormal human tissues. Am. J. Pathol. 150, 1443-1455 (1997)

7. Jackson, T. Efficient infection of cells in culture by type $O$ foot-and-mouth disease requires binding to cell surface heparan sulfate. /. Virol. 70, 5282-5287 (1996)

8. Rey, F.A., Heinz, F.X., Mandl, C., Kunz, C. \& Harrison, S.C. The envelope glycoprotein from tickborne encephalitis virus at $2 \AA$ resolution. Nature 375, 291-298 (1995)

9. Rothwell, S.W., Putnak, R. \& La Russa, V.F. Dengue2 virus infection of human bone marrow: characterization of dengue- 2 antigen-positive stromal cells. Am. J. Trop. Med. Hyg. 54, 503-510 (1996)

10. Kontny, U., Kurane, I. \& Ennis, F.A. Gamma interferon augments $\mathrm{Fc}$ gamma receptor-mediated dengue virus infection of human monocytic cells. ). Virol. 62, 3928-3933 (1988)

Department of Virus Diseases

Walter Reed Army

Institute of Research

Washington, DC 20307, USA

\title{
Immunostimulatory DNA: A clear and present danger?
}

\author{
Two recent studies demonstrate how sequence motifs in bacterial DNA can activate the immune system, \\ causing both beneficial as well as deleterious consequences (pages 849-854).
}

S DNA DANGEROUS? Among all biological molecules, this question seems the most incongruous when posed for DNA. Since the discovery of the double helix, DNA has occupied a unique status among macromolecules as an unequivocal source of good. DNA is the genetic blueprint and it is synonymous with life. To the extent that DNA causes harm, the problem has been considered external resulting from mutagens, carcinogens and an occasional mistake by the replication machinery. Furthermore, according to dogma that prevailed for almost 40 years, the beneficence of DNA extended to the immune system. Numerous studies suggested that DNA is immunologically inert, with an immune response to DNA emblematic of the profound distur-

\section{DAVID S. PISETSKY}

bances that provoke autoimmunity.

Investigations in a variety of disciplines have overturned this concept and have shown that DNA has a potential for danger. This potential reflects the existence of another genetic code, distinct from the triplets that encode amino acid sequences. This other genetic code distinguishes eukaryotic and prokaryotic DNA and, in binary fashion, alerts the immune system to infection ${ }^{1}$. In current terminology, this code is a danger signal ${ }^{2}$.

Unlike the classic genetic code, the danger code is based on short sequence motifs the content of which differs in eukaryotic and prokaryotic DNA. These se- quences bear the general structure of two 5' purines, an unmethylated CpG motif, and two $3^{\prime}$ pyrimidines $^{3-4}$. These sequences, termed immunostimulatory sequences (ISS) or CpG motifs, occur much more commonly in bacterial than in mammalian DNA for at least two reasons. The $\mathrm{C}$ and $\mathrm{G}$ bases occur in tandem much less commonly in mammalian DNA than predicted by base composition, a phenomenon known as CpG suppression. Furthermore, in mammalian DNA, cytosine is commonly methylated at the $\mathrm{C} 5$ position.

Because of its ISS content, bacterial DNA displays powerful immunological activities. These include induction of cytokines (IL-12, TNF- $\alpha$, IL-6, IFN- $\alpha / \beta$, IFN$\gamma, \mathrm{IL}-18)$, polyclonal $\mathrm{B}$ cell activation as 